\title{
Prediction Model of Coating Growth Rate for Varied Dip-Angle Spraying Based on Gaussian Sum Model
}

\author{
Yong Zeng, ${ }^{1}$ Yakun Zhang, ${ }^{1}$ Junxue He, ${ }^{2}$ Hai Zhou, ${ }^{1}$ Chunwei Zhang, ${ }^{1}$ and Lei Zheng ${ }^{1}$ \\ ${ }^{1}$ School of Mechanical Engineering, Yancheng Institute of Technology, Yancheng, Jiangsu 224051, China \\ ${ }^{2}$ College of Electrical and Information Engineering, Lanzhou University of Technology, Lanzhou, Gansu 730050, China \\ Correspondence should be addressed to Yong Zeng; zengzhong188@126.com
}

Received 27 September 2016; Accepted 15 November 2016

Academic Editor: Alessandro Gasparetto

Copyright (c) 2016 Yong Zeng et al. This is an open access article distributed under the Creative Commons Attribution License, which permits unrestricted use, distribution, and reproduction in any medium, provided the original work is properly cited.

\begin{abstract}
In automatic spraying of spray painting robot, in order to solve the problems of coating growth rate modeling for varied dipangle spraying technology, a prediction mode of coating growth rate using the Gaussian sum model is proposed. Based on the Gaussian sum model, a theoretical model for coating growth rate with varied dip-angle spraying is established by using the theory of differential geometry. The coating thickness of the sample points in the distribution range of the coating was obtained by making the experiment of varied dip-angle spraying. Based on the theoretical model, the nonlinear least square method is used to fit the coating thickness of the sample points and the parameter values of the theoretical model are calculated. By analyzing the variation law of the parameters with the spray dip-angle, the prediction model of coating growth rate for varied dip-angle spraying is established. Experiments have shown that the prediction model has good fitting precision; it can satisfy the real-time requirement with varied dip-angle spraying trajectory planning in the offline programming system.
\end{abstract}

\section{Introduction}

Trajectory planning of spray painting robot for complex free surface has been a hot research topic both at home and abroad. One of the key difficulties in the research of spraying trajectory planning is to establish a coating growth rate model with high accuracy and wide applicability [1-3]. At present, there are two main kinds of models for coating growth rate; a class of Gaussian distribution model $[4,5]$ and Cauchy distribution model [6] were proposed by Antonio and Freund, both of them belong to an infinite range model, and this kind of model is only suitable for the spray gun perpendicular to the surface of the workpiece. There is also limited range models, for example, piecewise function model [7], $\beta$ distribution model [8], analytical deposition model [9], parabolic model [10], ellipse dual- $\beta$ model [11], and Gaussian sum model [12]. The mathematical expressions of these models are usually derived by means of mathematical analysis. At the same time, based on the mathematical expressions, the final model of coating growth rate is approximated by the fitting of the experimental data. These models can be widely used in the case of complicated surface shape if these models meet the error requirement. In these models, the Gaussian sum model has a wide range of application; it can be applied to many kinds of spray gun and its fitting precision is higher.

In this paper, a prediction model of coating growth rate for varied dip-angle spraying based on the Gaussian sum model is established by the theory study and spraying experiment. The model can be used to generate the coating growth rate model under a certain spray dip-angle quickly and accurately.

\section{Theoretical Modeling of the Coating Growth Rate for Varied Dip-Angle Spraying}

Spraying process is the coating produced by the spray gun that atomize to the surface of the workpiece; the process is rather complicated. Under the action of the spray gun and the pressure vessel, the paint can realize the atomization and it is sprayed from the spray gun nozzle; the paint is formed in a conical or ellipse-conical shape in space. In this paper, the coating growth rate model is established for varied dipangle spraying based on a kind of spray gun, the paint space 


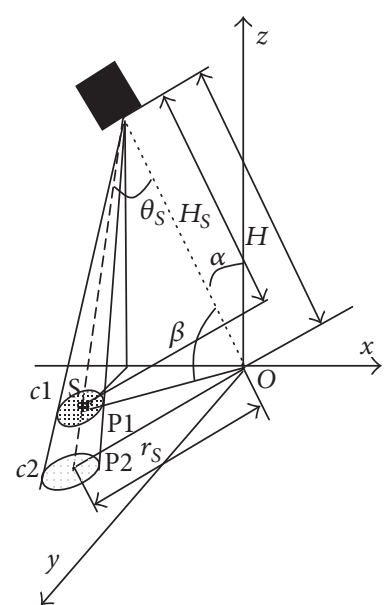

(a) Schematic diagram of the dipangle spraying calculation

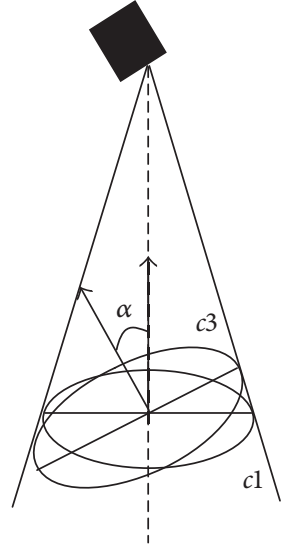

(b) Normal deflection angles of $c 1-c 3$
FIGURE 1: Schematic diagram of the coating growth rate model calculation for dip-angle spray painting.

distribution of the spray gun is conical, and the torch of the spray gun is a circular cross section and isotropic. Under the assumptions that the spray distance $H$, the spray flow $q_{v}$, and the cone angle $\varphi$ are constant when the spray gun is still sprayed, a mathematical model is used to describe the distribution of spray coating on a flat plate. The Gaussian sum model has the advantage of high fitting precision and not affected by the shape of the coating distribution, so it is assumed that the coating on the flat plate is distributed by the Gaussian sum model; its expression is

$$
f\left(r_{S}\right)= \begin{cases}\sum_{i=1}^{N} \omega_{i} \exp \left[\frac{-\left(r_{S}-r_{i}\right)^{2}}{2 \sigma_{i}^{2}}\right] & \left|r_{S}\right| \leq R \\ 0 & \left|r_{S}\right|>R\end{cases}
$$

where $\omega_{i}, r_{i}$, and $\sigma_{i}$ are the unknown parameters, $i=$ $1,2, \ldots, N ; R$ is the radius of spray painting. When $N$ tends to infinity, the function can approximate any form of coating distribution [13], but its complexity has increased dramatically, so generally take $N=3$.

In consideration of the dip-angle spraying, assume that the angle between the axis of the spray gun and the plane normal direction is $\alpha$, as the spray dip-angle. Using the area amplification theorem of differential geometry, assuming that the small round regions are $c 1$ and $c 2$ by spraying, respectively, in the reference planes $\mathrm{P} 1$ and $\mathrm{P} 2$ which are perpendicular to the spray direction of the spray gun, as shown in Figure 1(a), the areas of $c 1$ and $c 2$ are $S_{\mathrm{P} 1}$ and $S_{\mathrm{P} 2}$ respectively, and the corresponding coating thicknesses are $f_{1}$ and $f_{2} . H_{S}$ is the distance from any point $S$ on the plane to the nozzle along the spray gun axis. Therefore,

$$
\frac{S_{\mathrm{P} 1}}{S_{\mathrm{P} 2}}=\frac{H_{S}^{2}}{H^{2}} .
$$

So the coating thicknesses of $f_{1}$ and $f_{2}$ are satisfied:

$$
\frac{f_{1}}{f_{2}}=\frac{H^{2}}{H_{S}^{2}} \text {. }
$$

Suppose there are two plane regions at the same cone angle, respectively, which are $c 1$ and $c 3$, as shown in Figure 1(b), where $c 1$ is perpendicular to the spray direction; the angle between $c 3$ and the spray direction is $\alpha$, so the coating thickness of $c 3$ can be expressed as

$$
f_{3}=f_{1} \cos \alpha .
$$

Combining formulas (2)-(4), the theoretical model of coating growth rate which takes into account the spray dip-angle as a variable can be expressed as

$$
f(x, y, \alpha)=f\left(r_{S}\right)\left(\frac{H}{H_{S}}\right)^{2} \cos \alpha,
$$

where

$$
\begin{aligned}
H_{S} & =H+x \sin \alpha, \\
r_{S} & =\frac{H \sqrt{x^{2} \cos ^{2} \alpha+y^{2}}}{H+x \sin \alpha} .
\end{aligned}
$$

$x$ and $y$ are the horizontal and vertical coordinates of any point $S$ within the coating range.

\section{Fitting Method of Coating Growth Rate Model}

To establish the coating growth rate model under the prescribed dip-angle spraying, unknown parameters in formula (5) need to be solved further. Coating thickness data of the sample point is measured in the coating distribution range by doing the spraying experiment of the dip-angle. Fitting these data with the theoretical model by the least square method, unknown parameters can be obtained.

The rectangular coordinate system $X O Y$ is established with the intersection point $O$ of the spray gun axis and the spraying plane as the coordinate origin. Since the coating is formed on the plane after the dip-angle spraying, the coating covering range shape is elliptical. Here a rectangle is used to contain the ellipse; the length and width of the rectangle are the long and short axis of the ellipse. Isometric parallel lines parallel to the $X$-axis and the $Y$-axis are, respectively, done in the rectangular box; the intersection point between parallel lines is the sampling point of coating thickness, as shown in Figure 2. $M$ sampling points in the measurement range of coating thickness are measured; optimization function is established by using nonlinear least square method, as shown in the following:

$$
\begin{aligned}
& E\left(\omega_{1}, \omega_{2}, \omega_{3}, r_{1}, r_{2}, r_{3}, \sigma_{1}, \sigma_{2}, \sigma_{3}\right) \\
& =\min \sum_{i=1}^{M}\left[T\left(x_{i}, y_{i}\right)-f\left(x_{i}, y_{i}\right)\right]^{2},
\end{aligned}
$$




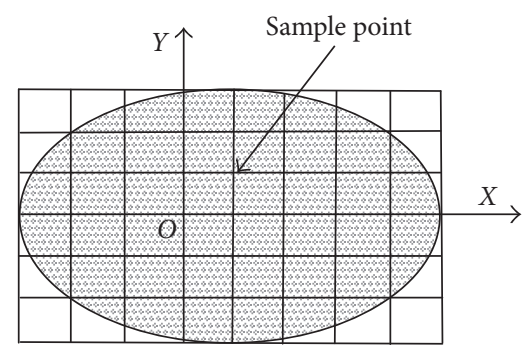

FIGURE 2: Sample points of coating thickness.

where $E$ is the sum of squares of the difference between the coating thickness of the sampling point and the theoretical calculation value; $T\left(x_{i}, y_{i}\right)$ is the coating thickness of the sampling point; $f\left(x_{i}, y_{i}\right)$ is the theoretical value calculated by formula (5).

In this study, the optimization algorithm of LevenbergMarquardt is used to solve the unknown parameter values; its basic steps are as follows:

(1) Determine the initial values of $p, \mu, v$, and $\varepsilon$.

(2) Calculate error matrix, corresponding Jacobi matrix $J$, and module $F$ of error matrix.

(3) Calculate Hessian matrix and gradient $g$.

(4) Calculate step size $m$ and determine whether $F$ is less than convergence value $\varepsilon$; if yes then the iteration ends; otherwise, go to the next step.

(5) Calculate $p=p+m$ and gain ratio $\rho$.

(6) Determine whether $\rho$ is greater than 0 and update iteration value.

(7) Determine whether $F$ is less than convergence value $\varepsilon$; if yes then the iteration ends; otherwise, go to the step (4).

The above algorithm is used to obtain a series of unknown parameter values of the corresponding coating growth rate model of spray dip-angle by using the finite number of varied dip-angle spraying experiments. With spray dip-angle as variable, the value of these parameters is analyzed with the change of spray dip-angle. Finally, a prediction model of coating growth rate for varied dip-angle spraying is established.

\section{Application of Prediction Model of Coating Growth Rate for Varied Dip-Angle Spraying}

In order to ensure the coating uniformity on the insidecorner surface and reduce the waste of paint on the outsidecorner surface, the continuous varied dip-angle spray trajectory planning method can be used to design spray trajectory on the inside-corner or outside-corner surface, as shown in Figure 3. If the continuous varied dip-angle spray trajectory parameters were optimized on the inside and outside corners surface, the coating thickness model is needed to establish after the spray gun along the trajectory of the continuous varied dip-angle spraying. In theory, based on the prediction model of coating growth rate, the dynamic coating thickness model can be obtained by integrating it under a certain spraying dip-angle.

Assume the trajectory length of the continuous varied dip-angle spraying is $d$; spray gun to achieve the maximum dip-angle is $\alpha_{\text {th }}$. In order to reduce the complexity of the problem, the trajectory of the continuous varied dip-angle spraying is divided into $i$ segments by the discrete method, the spray dip-angle of each segment is $(1 / i) \alpha_{\mathrm{th}},(2 / i) \alpha_{\mathrm{th}}, \ldots, \alpha_{\mathrm{th}}$, respectively, and the walking speeds of the spray gun are $v_{1}, v_{2}, \ldots$, and $v_{i}$, respectively. At this time, the coating thickness model of each small segment after the spray painting on the point $S$ can be expressed as follows:

$$
\begin{array}{r}
T_{S}(x, y, j)=\frac{1}{v_{j}} \int_{((j-1) / i) d}^{(j / i) d} f\left(x, y, \frac{j}{i} \alpha_{\mathrm{th}}, \gamma_{j}\right) d z, \\
\quad j \in[1, i], j \text { is a positive integer, }
\end{array}
$$

where

$$
\gamma_{j}=\frac{H \sqrt{y^{2}+(x-z)^{2} \cos ^{2}\left((j / i) \alpha_{\mathrm{th}}\right)}}{H+(x-z) \sin \left((j / i) \alpha_{\mathrm{th}}\right)} .
$$

Based on formula (8), the dynamic coating thickness model of the whole continuous varied dip-angle spray trajectory can be obtained by the superposition; expression is as follows:

$$
T_{S}(x, y)=\sum_{j=1}^{i} T_{S}(x, y, j) .
$$

From the above formula, the dynamic coating thickness model is established by the superposition of coating thickness models of constant dip-angle spraying. According to the prediction model of coating growth rate for varied dip-angle spraying, the coating thickness model of constant dip-angle spraying can be established quickly and meet the demand of the offline programming system for rapid modeling of the constant dip-angle spraying after continuous varied dip-angle spraying; this is also an important reason for the establishment of the prediction model of coating growth rate.

\section{Experimental Verification}

The experimental platform is composed of an automatic spray gun, an air compressor, and a bracket of spray gun with adjustable dip-angle and height. The workpiece to be sprayed is a $460 * 400 \mathrm{~mm}$ steel plate. Adjust the spray height $H$ to be $200 \mathrm{~mm}$ and the cone angle $\varphi$ to be $28^{\circ}$. Spraying time is set to $1 \mathrm{~s}$; the coating thickness of fixed point is measured by thickness gauge after spraying is finished and the coating is completely dried.

Adjust the spray dip-angle and in turn $0^{\circ}, 10^{\circ}, 20^{\circ}, 30^{\circ}$, and $40^{\circ}$ static spraying experiment, with continuous spraying 3 times in each dip-angle, take a coating covering the best of the experimental sample piece to measure the coating thickness, and thickness gauge MC-2010A is used, as shown in Figure 4. The coating thickness of each sampling point was measured 3 times and average value was obtained as the coating thickness of the sampling point. 
TABLE 1: Parameter values of different spraying dip-angle for Gaussian sum model.

\begin{tabular}{lccccccccc}
\hline$\alpha\left(^{\circ}\right)$ & $\omega_{1}$ & $r_{1}$ & $\sigma_{1}$ & $\omega_{2}$ & $r_{2}$ & $\sigma_{2}$ & $\omega_{3}$ & $r_{3}$ & $\sigma_{3}$ \\
\hline 0 & 100.6 & 2.4 & 13.5 & -45.2 & 27.4 & 7.1 & 91.3 & 27.3 & 7.6 \\
10 & 102.5 & 2.1 & 12.2 & -81.6 & 27.6 & 6.7 & 126.5 & 27.2 & 8.1 \\
20 & 100.1 & 2.1 & 14.4 & -185.7 & 27.4 & 7.3 & 221.4 & 27.7 \\
30 & 101.0 & 2.2 & 13.5 & -302.8 & 27.3 & 7.5 & 342.4 & 26.9 & 7.8 \\
40 & 100.7 & 2.6 & 13.3 & -406.8 & 27.4 & 7.1 & 446.0 & 27.4 & 7.4 \\
\hline
\end{tabular}
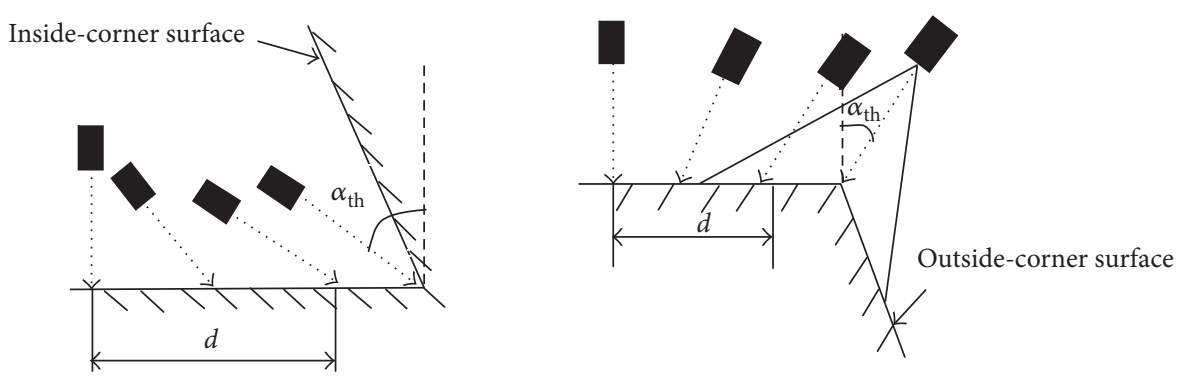

FIGURE 3: Sketch of continuous varied dip-angle spraying.
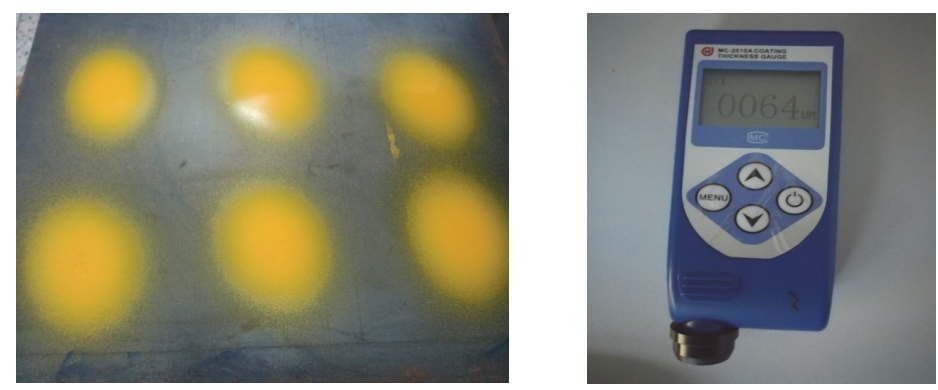

FIgURE 4: Sample piece and measuring tool.

After obtaining the coating thickness of the sampling point for each dip-angle spraying experiment, the unknown parameter values of the model were obtained for each spray dip-angle by using formula (7), as shown in Table 1.

By parameter values in Table $1, \omega_{2}$ and $\omega_{3}$ have obvious change with the spray dip-angle, the change law can be obtained by the curve fitting toolbox in MATLAB 2010, and the expressions are as follows:

$$
\begin{aligned}
& \omega_{2}(\alpha)=3952 \cos ^{2}(\alpha)-5446 \cos (\alpha)+1447, \\
& \omega_{3}(\alpha)=-3598 \cos ^{2}(\alpha)+4841 \cos (\alpha)-1151 .
\end{aligned}
$$

In addition to $\omega_{2}$ and $\omega_{3}$, the remaining 7 groups of parameters with the change of the dip-angle are not obvious. The reasons of the difference between these parameter values with the dip-angle are mainly caused by the measurement error of coating thickness and the calibration error of spray height and spray dip-angle. It is assumed that these parameter values are not related to the change of the dip-angle. The average values of each parameter are taken here, as follows:

$$
\begin{aligned}
\omega_{1} & =101, \\
r_{1} & =2.3, \\
\sigma_{1} & =13.4, \\
r_{2} & =27.4, \\
\sigma_{2} & =7.1, \\
r_{3} & =27.3, \\
\sigma_{3} & =7.7 .
\end{aligned}
$$

The prediction model of coating growth rate for varied dipangle spraying is established by formulas (11) and (12) into formula (5). In order to verify the validity of the prediction model, under the premise of ensuring that spray height, spray time, and cone angle are the same, spray experiments were 


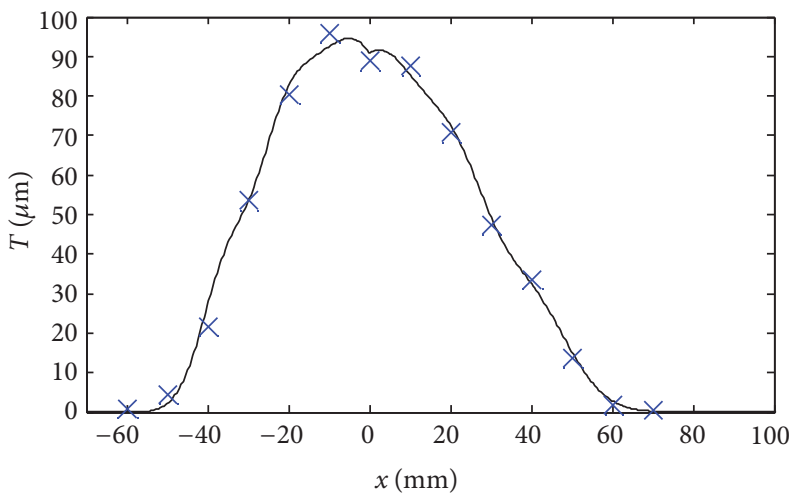

- Gaussian sum model

(a) $y=0$

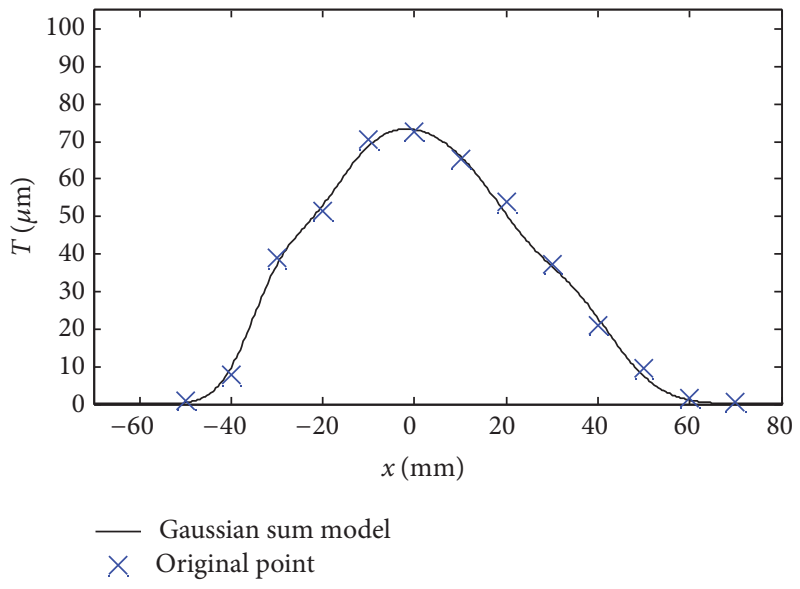

(c) $y=20$

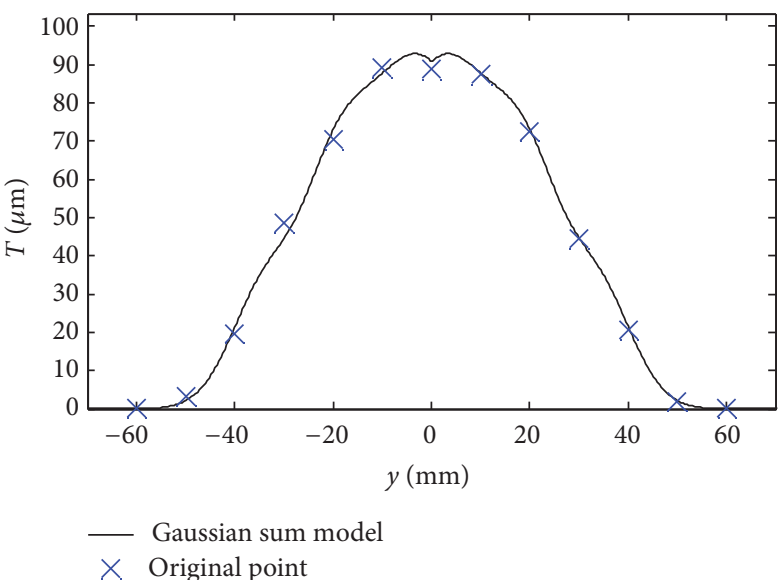

(b) $x=0$

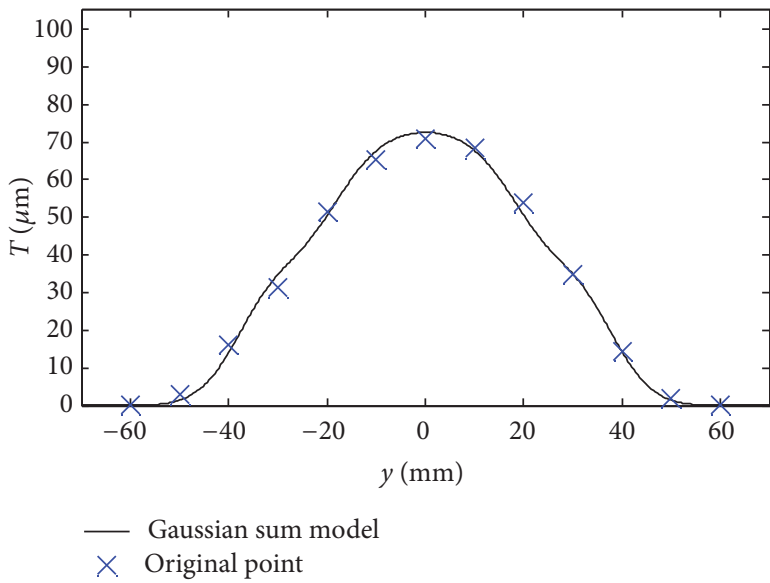

(d) $x=20$

FIGURE 5: Fitting of coating thickness when $\alpha=25^{\circ}$.

done when the spray dip-angle is $25^{\circ}$ and $45^{\circ}$, respectively. The coating thicknesses of the sample point were measured at $x=0, y=0, x=20 \mathrm{~mm}$, and $y=20 \mathrm{~mm}$. The coating thicknesses of the sampling point were compared with the prediction model, where Figure 5 shows the case when $\alpha=$ $25^{\circ}$ and Figure 6 shows the case when $\alpha=45^{\circ}$.

From Figures 5 and 6 , the prediction model can be used to well simulate the coating growth rate with varied dip-angle spraying; however, with the increase of spray dip-angle, the fitting precision of the prediction model has declined.

\section{Conclusion}

In this paper, in order to solve the modeling problem of coating growth rate for varied dip-angle spraying technology in the offline programming system of spray painting robot, the Gaussian sum model is used to establish the prediction model of coating growth rate for varied dip-angle spraying. Experiment shows that the Gaussian sum model also has a high fitting precision in describing the coating growth rate for varied dip-angle spraying, the prediction model can be used to reflect the coating growth rate quickly and accurately with varied dip-angle spraying, and it meets the requirements of offline programming system for modeling real-time performance and practicality. There are some advantages in the varied dip-angle spraying technology compared with the vertical spraying technology. For example, the varied dip-angle spraying technology can be a better way to ensure the coating uniformity on the inside-corner surface and reduce the waste of paint on the outside-corner surface. Based on the prediction model established in this paper, when spraying trajectory planning the surface of workpiece with inside and outsidecorner feature, a varied dip-angle spraying trajectory optimization model can be established to optimize the coating thickness uniformity in the corners of the surface, so as to achieve the purpose of the best spraying effect for the surface of workpiece with corner feature.

\section{Competing Interests}

The authors declare that they have no competing interests. 


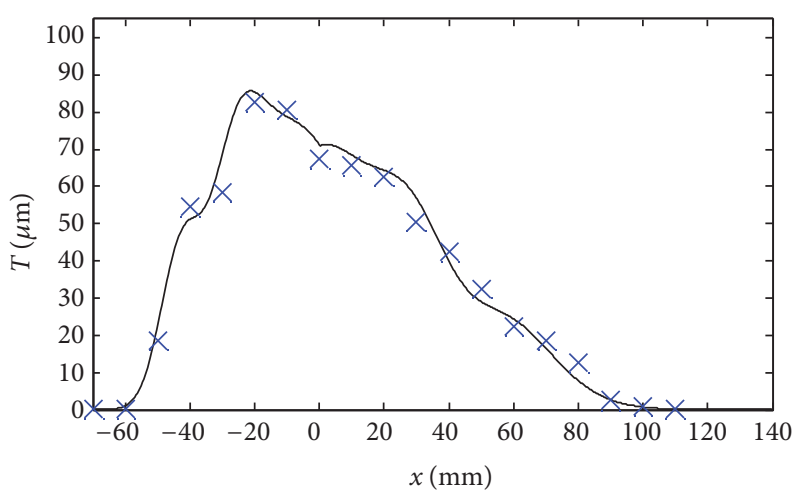

- Gaussian sum model $\times$ Original point

(a) $y=0$

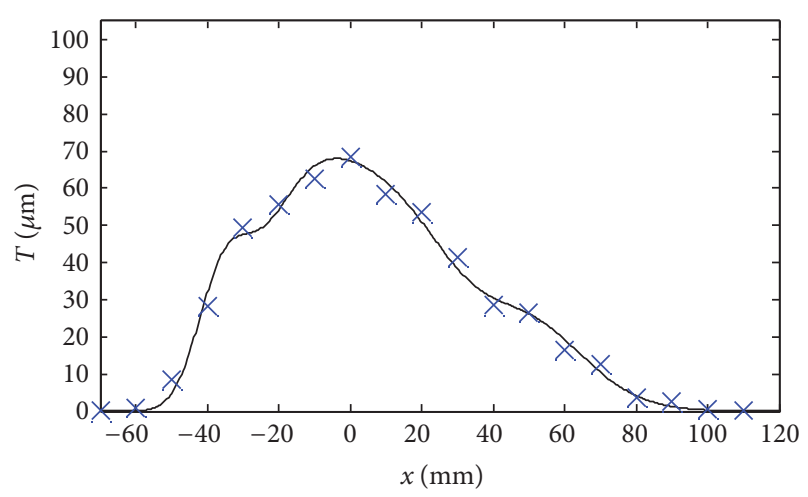

- Gaussian sum model

$\times$ Original point

(c) $y=20$

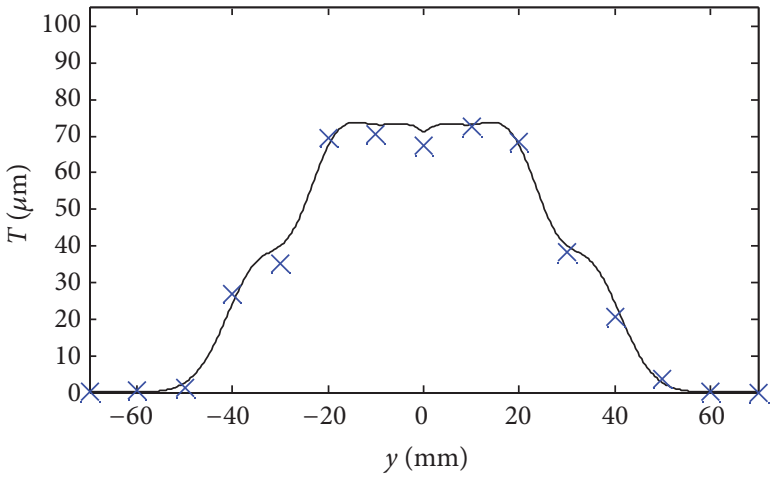

- Gaussian sum model

$\times$ Original point

(b) $x=0$

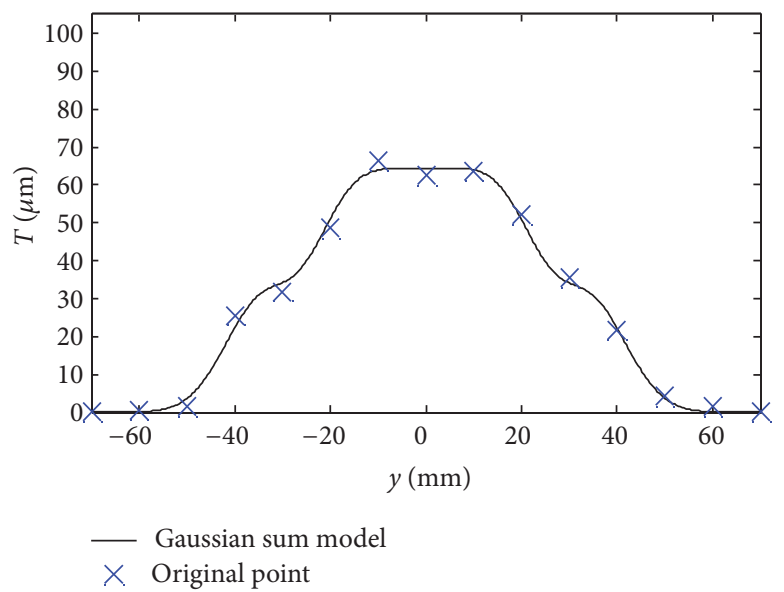

(d) $x=20$

FIGURE 6: Fitting of coating thickness when $\alpha=45^{\circ}$.

\section{Acknowledgments}

The project was supported by the National Natural Science Foundation of China (Grant no. 51405418) and the Natural Science Foundation of the Jiangsu Higher Education Institutions of China (Grant no. 14KJB460031).

\section{References}

[1] H. P. Chen and N. Xi, "Automated tool trajectory planning of industrial robots for painting composite surfaces," The International Journal of Advanced Manufacturing Technology, vol. 35, no. 7, pp. 680-696, 2008.

[2] H. P. Chen, T. Fuhlbrigge, and X. Z. Li, "A review of CAD-based robot path planning for spray painting," Industrial Robot, vol. 36, no. 1, pp. 45-50, 2009.

[3] H. P. Chen, N. Xi, S. K. Masood, Y. Chen, and J. Dahl, "Development of automated chopper gun trajectory planning for spray forming," Industrial Robot, vol. 31, no. 3, pp. 297-307, 2004.

[4] M. Hyotyiemi, "Minor moves-global results: robot trajectory planning," in Proceedings of the IEEE International Conference on Tools for Artificial Intelligence, pp. 16-22, Herndon, Va, USA, November 1990.
[5] E. Freund, D. Rokossa, and J. Rossmann, "Process-oriented approach to an efficient off-line programming of industrial robots," in Proceedings of the 24th Annual Conference of the IEEE Industrial Electronics Society, pp. 208-213, Los Alamitos, Calif, USA, 1998.

[6] J. K. Antonio, R. Ramabhadran, and T. L. Ling, "A framework for trajectory planning for automated spray coating," International Journal of Robotics and Automation, vol. 12, no. 4, pp. 124-134, 1997.

[7] C. Feng and Z.-Q. Sun, "Models of spray gun and simulation in robotic spray painting," Robot, vol. 25, no. 4, pp. 353-358, 2003.

[8] T. Balkan and M. A. S. Arikan, "Modeling of paint flow rate flux for circular paint sprays by using experimental paint thickness distribution," Mechanics Research Communications, vol. 26, no. 5, pp. 609-617, 1999.

[9] D. C. Conner, A. Greenfield, P. N. Atkar, A. A. Rizzi, and H. Choset, "Paint deposition modeling for trajectory planning on automotive surfaces," IEEE Transactions on Automation Science and Engineering, vol. 2, no. 4, pp. 381-391, 2005.

[10] T. Balkan and M. A. S. Arikan, "Surface and process modeling and off-line programming for robotic spray painting of curved surface," Journal of Robotic Systems, vol. 17, no. 9, pp. 479-494, 2000 . 
[11] Y. G. Zhang, Y. M. Huang, and F. Gao, "New model for air spray gun of robotic spray-painting," Journal of Mechanical Engineering, vol. 42, no. 11, pp. 226-233, 2006.

[12] B. Zhou, Z. H. Shao, Z. D. Meng, and X. Z. Dai, "Gaussian sum based coat growth rate modeling of spray painting robots," Journal of Huazhong University of Science and Technology (Natural Science Edition), vol. 41, supplement 1, pp. 463-466, 2013.

[13] C. E. Rasmussen and C. K. I. Williams, Gaussian Processes for Machine Learning, MIT Press, Cambridge, UK, 2006. 


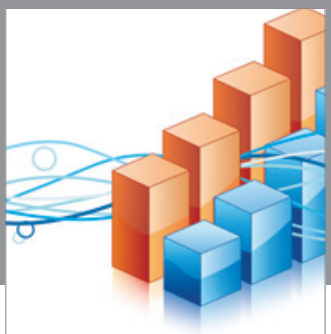

Advances in

Operations Research

vatem alat4

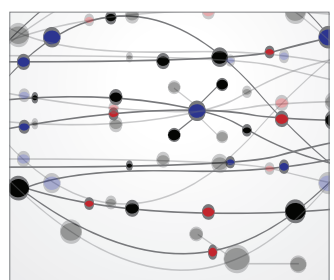

\section{The Scientific} World Journal
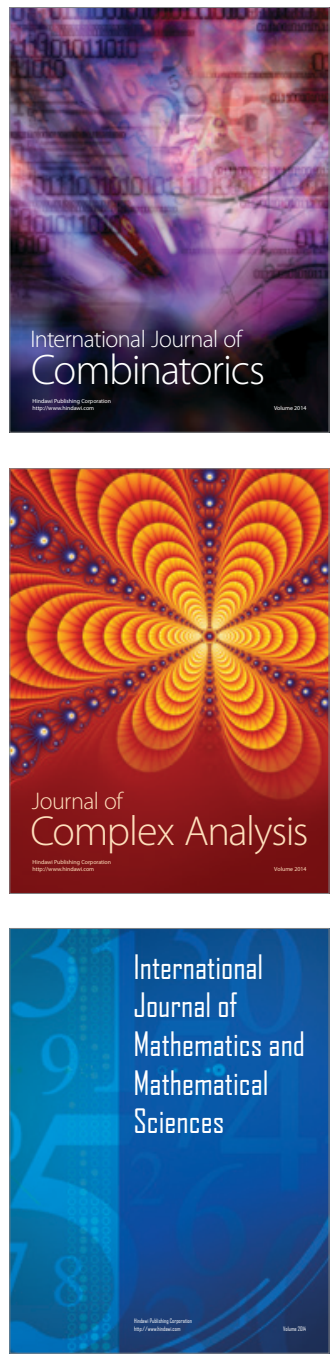
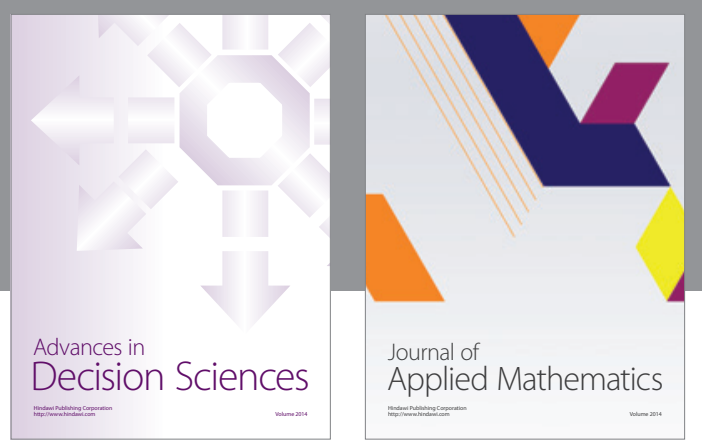

Algebra

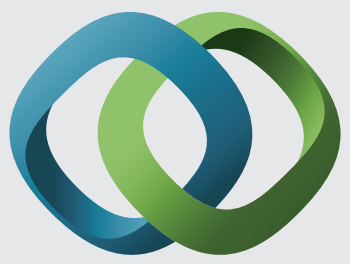

\section{Hindawi}

Submit your manuscripts at

http://www.hindawi.com
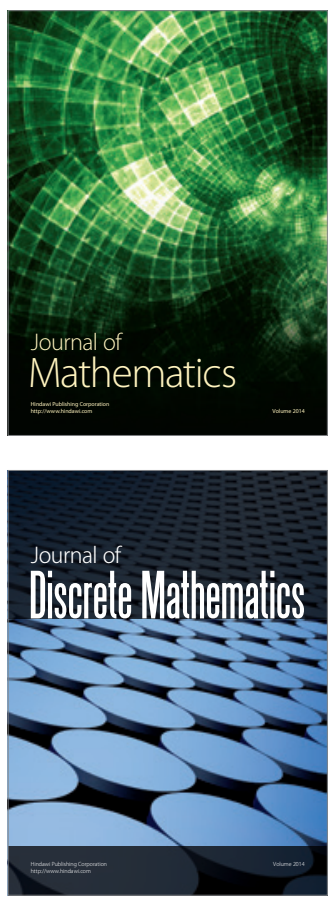

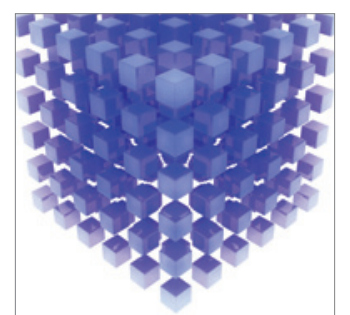

Mathematical Problems in Engineering
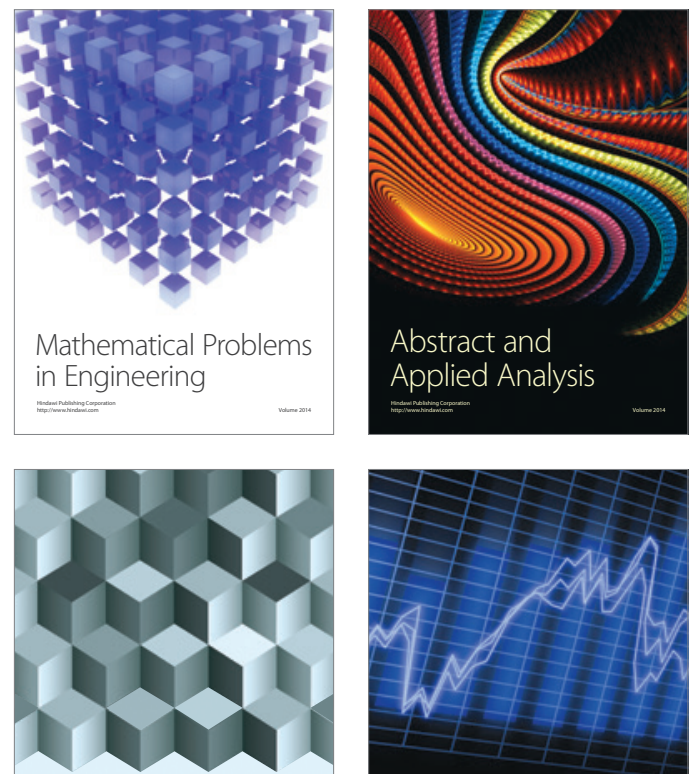

Journal of

Function Spaces

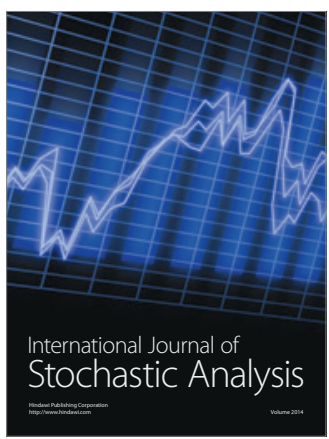

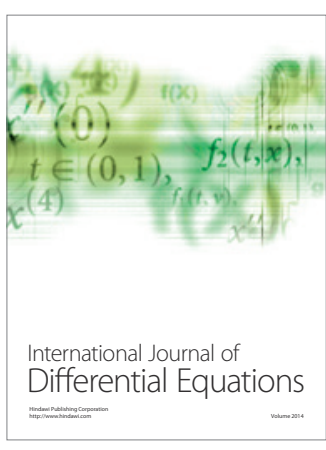
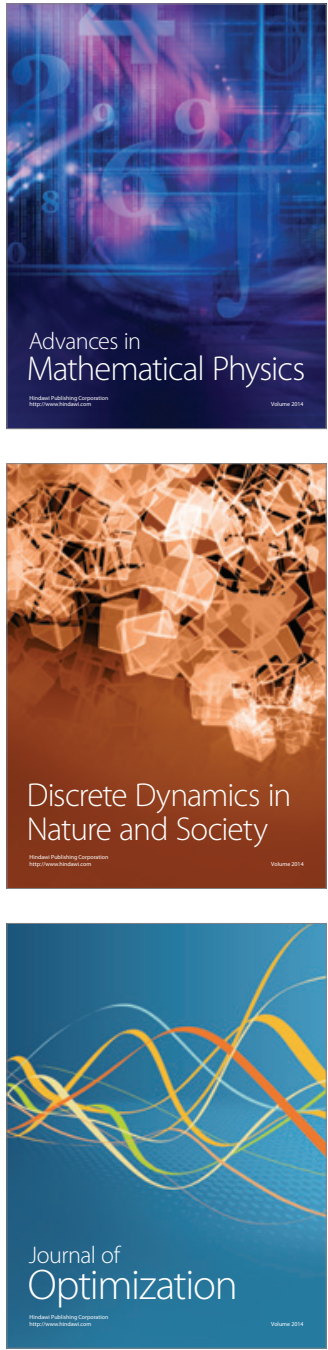